\title{
BMJ Open Health-related quality of life: population epidemiology and concordance in Australian children aged 11-12 years and their parents
}

\author{
Max Catchpool, ${ }^{\oplus 1}$ Lisa Gold, ${ }^{\oplus, 3}$ Anneke C Grobler, ${ }^{\oplus 3,4}$ Susan A Clifford, ${ }^{\oplus 3,4}$ \\ Melissa Wake $3,4,5$
}

To cite: Catchpool M, Gold L, Grobler AC, et al. Health-related quality of life: population epidemiology and concordance in Australian children aged $11-12$ years and their parents. BMJ Open 2019;9:157-164. doi:10.1136/ bmjopen-2018-022398

- Prepublication history for this paper is available online. To view these files, please visit the journal online (http://dx.doi. org/10.1136/bmjopen-2018022398).

Received 15 February 2018 Revised 24 May 2018 Accepted 4 April 2019

Check for updates

(C) Author(s) (or their employer(s)) 2019. Re-use permitted under CC BY-NC. No commercial re-use. See rights and permissions. Published by BMJ.

${ }^{1}$ Melbourne School of Population and Global Health, The University of Melbourne, Parkville, Victoria, Australia

${ }^{2}$ School of Health and Social Development, Deakin University, Geelong, Victoria, Australia ${ }^{3}$ Murdoch Children's Research Institute, Parkville, Victoria, Australia

${ }^{4}$ Department of Paediatrics, The University of Melbourne, Parkville, Victoria, Australia ${ }^{5}$ Department of Paediatrics and the Liggins Institute, The University of Auckland, Auckland, New Zealand

Correspondence to Professor Melissa Wake; melissa.wake@mcri.edu.au

\section{ABSTRACT}

Objectives To describe the distribution of health-related quality of life (HRQL) in a national sample of Australian children aged 11-12 years and their parents, and examine associations within parent-child dyads.

Design The Child Health CheckPoint, a populationbased cross-sectional study nested between waves 6 and 7 of the Longitudinal Study of Australian Children (LSAC).

Setting Assessment centres in seven Australian cities and eight regional towns, or home visit; February 2015 to March 2016.

Participants Of all participating CheckPoint families $(\mathrm{n}=1874), 1853$ children (49.0\% girls) and 1863 parents (87.7\% mothers) with HRQL data were included (1786 pairs).

Outcome measures HRQL was self-reported using preference-based (Child Health Utility 9Dimension, CHU9D) and non-preference-based (Pediatric Quality of Life, PedsQL V.4.0) measures for children and preferencebased measures for parents (CHU9D; Assessment of Quality of Life 8 Dimension, AQoL-8D). Utility scores from preference-based measures were calculated using existing Australian algorithms to present a score on a 0-1 scale, where 1 represents full health. Parent-child concordance was assessed using Pearson's correlation coefficients and adjusted linear regression models. Survey weights and methods were applied to account for LSAC's complex sample design, stratification and clustering within postcodes.

Results Children's means and SD were 0.81 (SD 0.16) for CHU9D and 78.3 (SD 13.03) for PedsQL. In adults, mean HRQL for AQOL-8D and CHU9D were 0.78 (SD 0.16) and 0.89 (SD 0.10), respectively. Mean HRQL was similar for boys and girls, but slightly higher for fathers than mothers. The Pearson correlation coefficient for parent-child CHU9D values was $0.13(95 \% \mathrm{Cl} 0.09$ to 0.18$)$. Percentiles and concordance are presented for both samples for males and females separately and together.

Conclusions We provide Australian paediatric population values for HRQL measures, and the first national CHU9D values for mid-life adults. At age 11-12 years in this relatively healthy sample, parent-child concordance in HRQL was small.
Strengths and limitations of this study

- This large cross-sectional study provides Australian reference data for future studies investigating health-related quality of life (HRQL).

- This is first study to identify the distribution of HRQL values using the Child Health Utility 9 Dimension (CHU9D) in preadolescent children and mid-life adults in Australia.

- This is also the first study to investigate concordance of preference-based HRQL in parent-child dyads, including both mother-child and father-child estimates.

- Our adult study sample contained a large proportion of mothers, limiting the precision of descriptive and concordance estimates for fathers.

- Families living in disadvantaged neighbourhoods were under-represented.

\section{INTRODUCTION}

Measurements of health-related quality of life (HRQL) using patient-rated questionnaires are commonly used when assessing the effectiveness and cost-effectiveness of healthcare interventions. HRQL can be summarised as a multidimensional construct that measures the impact of health or disease on physical and psychosocial functioning. ${ }^{1}$ Various HRQL instruments exist, their main differences being the way in which they describe health, the number and types of dimensions and whether they are preference or non-preference based. Non-preference-based measures provide equal weight to each dimension or weight dimensions simply by the number of items included within them. ${ }^{2}$ Preference-based measures use preference weights to assign relative importance to different items and/or dimensions. ${ }^{2}$ The weights are typically calculated based on the preferences of a representative sample of the general population. A single number is calculated and 
represents the respondents' health status at that point in time. Preference-based measures generally produce an overall score that ranges from a state of health equivalent to full or perfect health (1) to a state of health equivalent to being dead $(0)$, and often to states considered worse than death $(<0) .^{3}$ Generic preference-based measures are the most widely used mechanism to calculate quality-adjusted life years (QALYs). ${ }^{3}$ QALYs express the benefits of healthcare treatment and preventative programmes in a common metric and guide healthcare resource allocation decisions. The underlying strength of the QALY is that it facilitates comparison across diseases and conditions.

Most preference-based HRQL measures have been developed for adults. Multiple measures are available for use in adult populations, many with preference weights developed for Australia. ${ }^{4}$ The child HRQL literature is less developed, and researchers have been reluctant to transfer the principles and definitions of adult HRQL to child HRQL. ${ }^{5}$ Measuring HRQL in childhood is difficult due to the dynamic nature of physical, emotional and cognitive changes with development. ${ }^{6}$ The language and content of children's HRQL measures need to apply to a range of developmental stages.

A recent review conducted by Chen and Ratcliffe identified nine generic preference-based instruments available internationally for use in paediatric populations. ${ }^{7}$ Among these instruments, the Child Health Utility 9 Dimension (CHU9D) is a relatively newly developed paediatric generic preference-based measure of HRQL. ${ }^{8}$ It was developed in England, and has preference weights available for multiple countries, including Australia. ${ }^{9}$ The nine dimensions were derived from qualitative interviews with children aged 7-11 years with a wide range of acute and chronic health conditions. ${ }^{9}$ These dimensions (worried, sad, pain, tired, annoyed, schoolwork, sleep, daily routine and ability to join in activities) encapsulate attributes of health most relevant to children. The CHU9D can also be adapted for adult completion by rephrasing the school work domain as work. Since the development of Australian preference weights, the CHU9D's use in Australian children has mostly been limited to validation and condition-specific studies. ${ }^{10-13}$ Its use in adult samples is still preliminary in Australia and elsewhere (Katherine Stevens, personal communication, 2017). No national studies have reported CHU9D values for the entire Australian population. Population norms of HRQL are important as they provide a base level and can be used for comparison purposes with other samples.

Observational studies have shown that the HRQL of parents of children with chronic conditions are significantly below population norms. ${ }^{14}{ }^{15}$ Intervention studies have shown that paediatric interventions that improve child health can also improve parental HRQL. ${ }^{16}$ However, these studies have measured child and parent HRQL with different instruments and in disease-specific contexts; the association between child and parent HRQL in the general population is yet to be explored using comparable measures (Catchpool Major Report Master of Health
Economics, Deakin University, 2016). To determine concordance for HRQL the same instrument and scoring algorithm must be applied, otherwise any differences could be attributed to the different scoring techniques. If an association exists between child and parental HRQL, then this could provide useful information for health professionals and researchers in terms of increasing the focus on parental HRQL in child and adolescent health and on children's HRQL in adult health services.

The Longitudinal Study of Australian Children's (LSAC) Child Health CheckPoint offers a unique opportunity to examine these associations. We aimed to describe the distribution of HRQL in a population-representative sample of Australian children aged 11-12 years and their parents, and the concordance between parents and children.

\section{METHODS}

\section{Study design and participants}

Details of the initial LSAC study design and recruitment are outlined elsewhere. ${ }^{17} 18$ Briefly, LSAC recruited a nationally representative sample of 5107 Australian infants ${ }^{19}$ using a two-stage random sample design with postcode as the primary sampling unit, and has since conducted a further six biennial data collection waves. The initial recruitment rate in 2004 was $57.2 \%$, of whom $73.7 \%(\mathrm{n}=3764)$ were retained to LSAC wave 6 in 2014. During the wave 6 visit, families were introduced to the upcoming detailed cross-sectional biophysical assessment (the Child Health CheckPoint), and 3513 families consented to their contact details being shared with the CheckPoint team for the purpose of recruitment into the module. CheckPoint took place between February 2015 and March 2016 at child age 11-12 years. Data completeness was high and participants were included in the current analyses if they contributed any HRQL data that could be scored (figure 1). The scoring algorithm of each instrument specify how missing data are treated. The CHU9D required no missing data for a total score to be calculated. The Assessment of Quality of Life 8 Dimension (AQoL-8D) scoring algorithm allows a limited number of missing values be imputed within each of its dimensions. The Pediatric Quality of Life (PedsQL) allows two to four missing values in each subscale for a mean score to be calculated. Non-biological child-parent pairs were excluded from the concordance analyses. A more detailed description of the CheckPoint study design is available elsewhere. ${ }^{20-22}$

\section{Consent}

The attending parent/caregiver provided written informed consent for themselves and their child to participate in the study.

\section{Procedure}

Child and parent participants attended a 'pop-up' assessment centre for a $3 \frac{1}{2} 2$ hour (capital and large cities) 
LSAC Wave 1, 2004: $n=5107$

Attrition: $n=1343$

LSAC Wave 6, 2014: $n=3764$

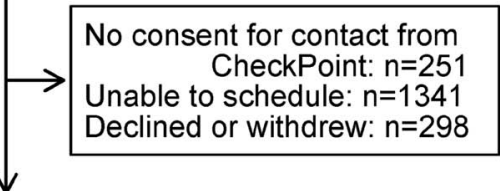

Child Health CheckPoint sample: $\mathrm{n}=1874$ (MAC $n=1356, m A C n=153, H V n=365$ )

Refused measure: $c=0, p=1$ Unable to assess: ${ }^{*} c=21, p=10$
Quality of Life data available for 1853 children and 1863 parents (1786 pairs)^

Figure 1 Participant flowchart. *Unable to assess due to equipment failure, poor quality data or time contraints. $\wedge$ Data from 16 non-biological child-parent pairs and 43 pairs for incomplete data in CHU9D utility measure excluded from concordance analyses. c, number of children; CHU9D, Child Health Utility 9 Dimension; HV, home visit assessment; LSAC, Longitudinal Study of Australian Children; MAC, main assessment centre; mAC, mini assessment centre; $n$, number of families; $p$, number of attending adults.

or $2^{1 / 2}$ hour (smaller regional centres) visits; those who were unable or declined to attend the centre were offered a 11/2 hour home visit (figure 1). At the assessment centres, participants rotated through various data collection stations every $15 \mathrm{~min}$. Throughout the visit, each participant completed a detailed questionnaire

Table 1 Sample characteristics, stratified by sex; values are weighted mean (SD), except where specified as \%

\begin{tabular}{llll}
\hline Characteristic & All & Male & Female \\
\hline $\begin{array}{llll}\text { Child } \\
\mathrm{n}\end{array}$ & 1853 & 945 & 908 \\
\hline Age, years & $12.0(0.4)$ & $12.0(0.4)$ & $12.0(0.4)$ \\
BMl, kg/m ${ }^{2}$ & $19.5(3.7)$ & $19.3(3.8)$ & $19.7(3.7)$ \\
BMl z-score & $0.37(1.09)$ & $0.34(0.99)$ & $0.39(0.99)$ \\
\hline $\begin{array}{l}\text { Disadvantage } \\
\text { index }\end{array}$ & $1009(62)$ & $1007(62)$ & $1010(62)$ \\
Parent & & & \\
n & 1863 & 229 & 1634 \\
Age, years & $43.6(5.6)$ & $46.4(7.1)$ & $43.3(5.3)$ \\
BMI, kg/m ${ }^{2}$ & $28.4(6.4)$ & $28.9(4.9)$ & $28.3(6.6)$ \\
\hline
\end{tabular}

BMI, body mass index; Disadvantage index, index of relative socioeconomic disadvantage; $n$, number of participants. administered on an iPad using the Research Electronic Data Capture (REDCap) tool ${ }^{23}$ during waiting periods. This included the HRQL measures that are the topic of this paper.

\section{Outcome measures}

Each respondent completed two self-rated HRQL measures as part of a larger questionnaire. The children's questionnaires were the preference-based CHU9D and the non-preference-based PedsQL V.4.0 measures. The adult measures were both preference-based measures, the CHU9D and the AQoL-8D.

The CHU9D is a paediatric generic preference-based measure of HRQL. It consists of a nine-item descriptive system and a set of preference weights, giving utility values for each health state described by the descriptive system, allowing the calculation of QALYs for use in cost utility analysis. ${ }^{9}$ Scoring algorithms for the CHU9D have been updated between publications and differ between countries. Although the CHU9D descriptive system was developed for young people, the original scoring algorithm for the CHU9D was based on adult general population values using the standard gamble method. More recently, Australian adolescent and adult scoring algorithms were developed using best worst scaling methods. ${ }^{9}$ Both techniques generate a score, that is, on the range of equivalence from perfect health (1) to being dead (0). To assess the distribution of HRQL data using the CHU9D, the adolescent scoring algorithm was applied to child data and the adult scoring algorithm on the adult data. However, to assess concordance of HRQL among child-parent dyads using the CHU9D, a common scoring algorithm needed to be applied. If this does not occur, any differences between HRQL of children and HRQL of their parents may be because of the different scoring methods used. Therefore, the adolescent algorithm was also applied to the parent CHU9D data, solely for the purposes of the intergenerational concordance analysis of this study.

The PedsQL is a standardised generic assessment instrument with versions that assess children's and parent's perception of child HRQL. ${ }^{24}$ Children aged 5-18 years can complete the child self-report section of the questionnaire. Unlike the CHU9D, the PedsQL is not preference based and each of its 23 items have an equal weight in calculating the total score. ${ }^{24}$ Therefore, the PedsQL cannot be used directly in the calculation of a QALY. US population normative data indicate high levels of internal consistency. ${ }^{25}$ In this study, the Generic Core Scale score from the PedsQL V.4.0 is reported.

The AQoL-8D contains 35 items and comprises eight separately scored dimensions, consisting of independent living, relationships, mental health, coping, pain, senses, self-worth and life satisfaction. ${ }^{26}{ }^{27}$ Like the CHU9D, the AQoL-8D is a multiattribute utility instrument. This means that there is a separate utility scoring algorithm allowing the calculation of utility values for each participant and 
Table 2 HRQL measures in Australian children and parents

\begin{tabular}{|c|c|c|c|c|c|c|c|c|c|c|c|c|}
\hline \multirow[b]{2}{*}{ HRQL measure } & \multicolumn{4}{|l|}{ All } & \multicolumn{4}{|c|}{ Males } & \multicolumn{4}{|c|}{ Females } \\
\hline & $n$ & Mean & SD & $95 \% \mathrm{Cl}$ & $n$ & Mean & SD & $95 \% \mathrm{Cl}$ & $\mathbf{N}$ & Mean & SD & $95 \% \mathrm{Cl}$ \\
\hline \multicolumn{13}{|l|}{ Children } \\
\hline Adolescent algorithm & 1827 & 0.81 & 0.16 & 0.81 to 0.82 & 931 & 0.81 & 0.16 & 0.80 to 0.83 & 896 & 0.82 & 0.15 & 0.81 to 0.83 \\
\hline PedsQL & 1847 & 78.3 & 13.0 & 77.6 to 79.0 & 942 & 77.6 & 12.7 & 76.6 to 78.6 & 905 & 79.1 & 13.4 & 78.0 to 80.2 \\
\hline \multicolumn{13}{|l|}{ Parents } \\
\hline Adult algorithm & 1843 & 0.89 & 0.10 & 0.88 to 0.90 & 227 & 0.91 & 0.08 & 0.90 to 0.92 & 1616 & 0.89 & 0.10 & 0.88 to 0.89 \\
\hline Adolescent algorithm & 1843 & 0.81 & 0.18 & 0.80 to 0.82 & 227 & 0.84 & 0.16 & 0.82 to 0.87 & 1616 & 0.80 & 0.18 & 0.79 to 0.82 \\
\hline AQoL-8D & 1861 & 0.78 & 0.16 & 0.77 to 0.79 & 229 & 0.80 & 0.14 & 0.78 to 0.83 & 1632 & 0.78 & 0.16 & 0.77 to 0.79 \\
\hline
\end{tabular}

AQoL-8D, Assessment of Quality of Life 8 Ddimension; CHU9D, Child Health Utility 9 Dimension; HRQL, health-related quality of life; $n$, number of participants in cohort with this measure (denominator); PedsQL, Pediatric Quality of Life.

QALYs. The utility algorithm used in the current study is derived from the Australian general population. ${ }^{26}$

\section{Potential confounders}

Body mass index

Recent evidence indicates that primary school students' adjusted mean utilities for HRQL are lower for overweight or obese students than healthy-weight students. ${ }^{12}$ Body mass index (BMI) was calculated from measured height and weight values $\left(\mathrm{kg} / \mathrm{m}^{2}\right)$. For children, $\mathrm{z}$ score was calculated according to US Centers for Disease Control reference values ${ }^{28}$ using the Stata 'zanthro' function.

\section{Socioeconomic indexes for areas}

Neighbourhood socioeconomic position is a potential confounder of HRQL. ${ }^{29}$ We used the socioeconomic indexes for areas (SEIFA) index of relative socioeconomic disadvantage (disadvantage index) score, a standardised score by geographic area (in this case, postcode of residence) compiled from 2011 Australian census data. The disadvantage index numerically summarises the social and economic conditions of Australian neighbourhoods (national mean of 1000 and a SD of 100, where higher values represent less disadvantage).$^{30}$

Child date of birth (DOB) and sex were imported into LSAC from Medicare Australia's enrolment database. Attending parent DOB was self-reported by parent questionnaire. Age was calculated to nearest week from the participant's DOB and date of assessment. Parent sex was self-reported.

\section{Statistical analysis}

Data were analysed using Stata V.14.2. HRQL measures were described for all children and adults (ie, regardless of relationship to child) for males and females separately and combined, using means and SD, percentiles and density plots. Population summary statistics and proportions were estimated by applying survey weights and survey procedures that corrected for sampling, participation and non-response biases, and took into account clustering in the sampling frame. SEs were calculated taking into account the complex design and weights. ${ }^{31}$ More detail on the calculation of weights is provided elsewhere. ${ }^{20} 32$

Concordance between HRQL of parents and HRQL of children for the CHU9D was assessed using (1) Pearson's correlation coefficients with 95\% CIs and (2) linear regression with the child $\mathrm{HRQL}$ as the dependent variable and parent HRQL as the independent variable, adjusted for parent age, child age, disadvantage index, as well as parent and child sex in models including both sexes. These same analyses were repeated using weighted multilevel survey analyses. As these results were similar, only the unweighted results are reported for concordance. As a sensitivity analysis, we repeated all analyses using child and adult CHU9D data valued with the adult algorithm.

\section{Patient and public involvement}

Because LSAC is a population-based longitudinal study, no patient groups were involved in its design or conduct. To our knowledge, the public was not involved in the study design, recruitment or conduct of the LSAC study or its CheckPoint module. Parents received a summary health report for their child and themselves at or soon after the assessment visit. They consented to take part knowing that they would not otherwise receive individual results about themselves or their child.

\section{RESULTS}

\section{Sample characteristics}

Of the 1874 families who participated, at least one HRQL measure was available for 1853 children $(98.9 \%)$ and 1863 parents (99.4\%) (figure 1), and 1786 parent-child dyads. There were slightly more boys $(n=945,51 \%)$ than girls $(\mathrm{n}=908,49 \%)$ and the parent sample was predominantly mothers $(\mathrm{n}=1634,87.7 \%)$ from a relatively socioeconomically advantaged position. The sample average disadvantage index score was slightly above the national average and showed a narrower distribution, reflecting a 


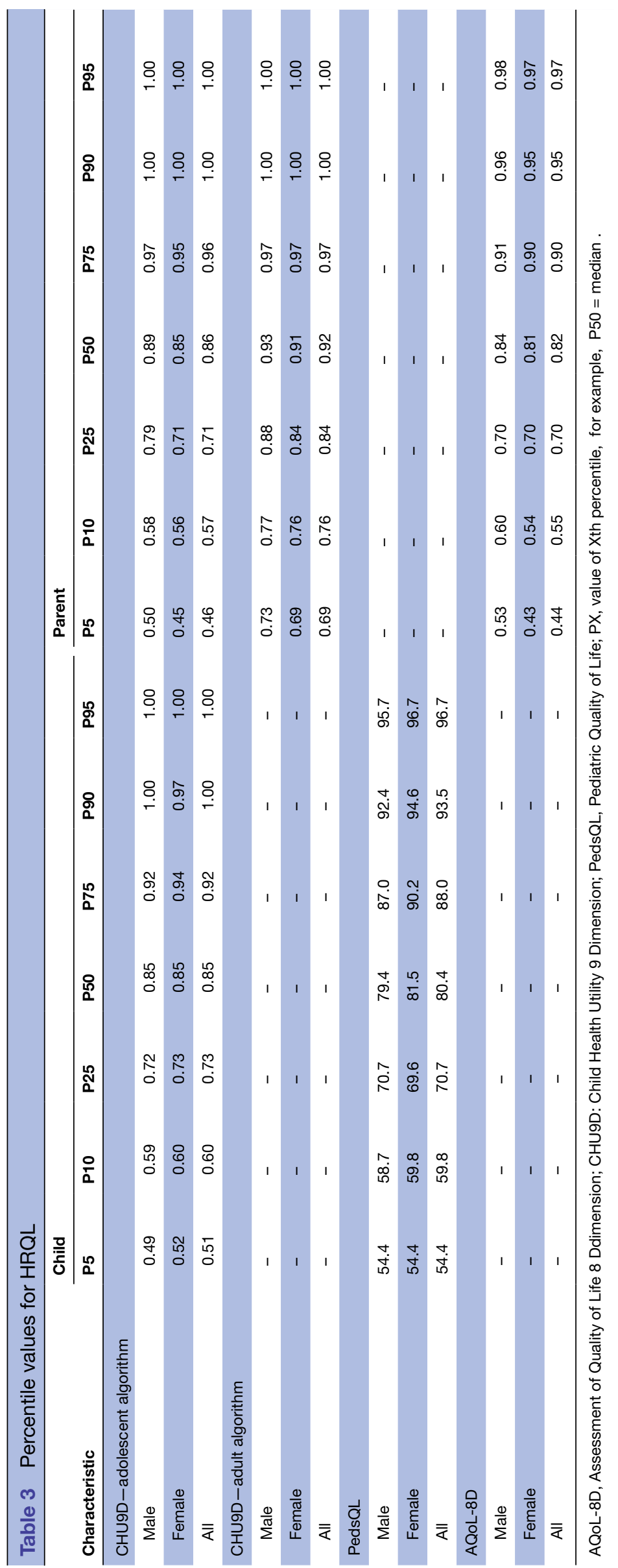

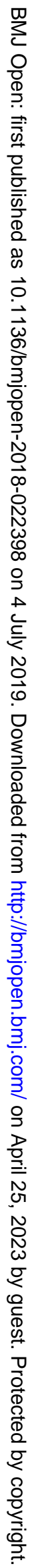



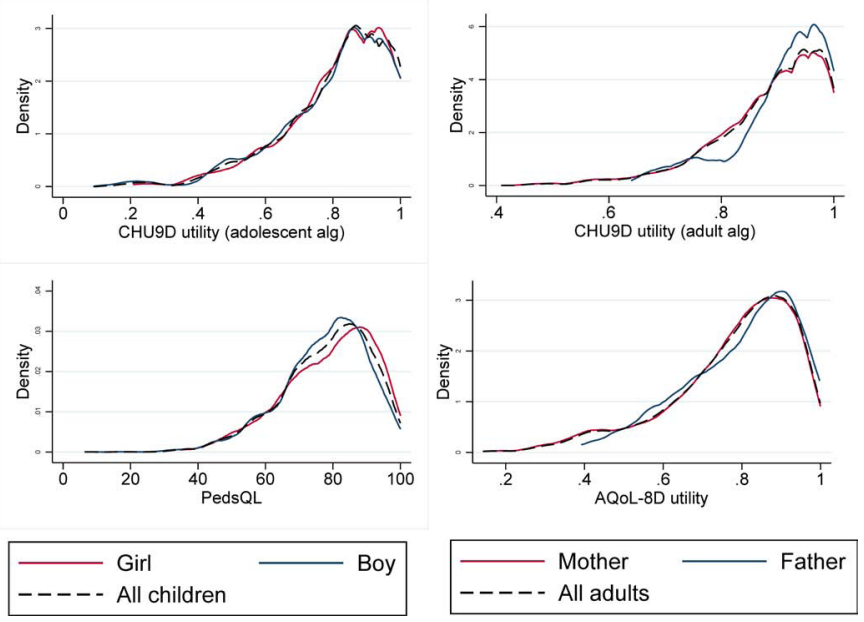

Figure 2 Distribution of HRQL in children and parents. Red line: girls/mothers; blue line: boys/fathers; black, dotted line: all. AQoL-8D, Assessment of Quality of Life 8 Dimension; CHU9D, Child Health Utility 9 Ddimension; HRQL, healthrelated quality of life; PedsQL, Pediatric Quality of Life.

lower representation of socioeconomically disadvantaged families in this sample (table 1).

Other participant characteristics were broadly representative of the Australian population.

\section{Distributions of HRQL}

\section{Children}

The mean CHU9D score for children was 0.81 (SD 0.16) (table 2). Extended percentile values (from 5th to 95th) are provided for all measures in table 3 , showing a median of 0.85 (IQR 0.73 to 0.92 ).

Values for boys and girls were largely similar. The mean PedsQL was 78.3 (SD 13.0), with a slight variation between boys (77.6, SD 12.7) and girls (79.1, SD 13.4). The distribution of HRQL was skewed, with most children reporting high HRQL and smaller numbers in worse health states (figure 2). This distribution was similar for boys and girls and similar across preference-based and non-preference-based measures.
Adults

The mean score of CHU9D in Australian mid-life adults was 0.89 (SD 0.10). Values using the CHU9D adolescent algorithm (as required for concordance analysis) were somewhat lower, due to the difference in item weights between the adult and adolescent scoring algorithms, with a mean CHU9D score for adults of 0.81 (SD 0.18). The AQoL-8D had a mean score across all parents of $0.78(0.16)$. For all HRQL measures in adults, scores were slightly higher in men than women. The distribution of HRQL was skewed to the left for both the preference-based measures used with parents, with slight differences between men and women (figure 2).

\section{Intergenerational concordance of HRQL}

A weak positive correlation was present between child HRQL and parent HRQL as measured by the CHU9D (correlation coefficients for Pearson 0.13, 95\% CI 0.09 to 0.18) (table 4). Results from the sensitivity analysis (using adult instead of adolescent algorithm for the parent CHU9D) were similar $(0.15,95 \%$ CI 0.10 to 0.19$)$. This mainly reflected mother-child concordance; there was no convincing evidence of father-child concordance. Coefficients were also very similar in the adjusted linear regression models after adjusting for potential confounders and demographic characteristics.

\section{DISCUSSION}

\section{Principal findings}

We have described HRQL values for Australian children aged 11-12 years and their parents, together with the first reported concordance within parent-child dyads. The results of our study provide normative data for three separate HRQL instruments that we hope will be useful to researchers who intend to use these instruments. In this generally healthy population, there was only a weak intergenerational concordance for HRQL.

Table 4 Child-parent concordance on the CHU9D adolescent version

\begin{tabular}{|c|c|c|c|c|c|c|c|c|c|}
\hline \multirow[b]{2}{*}{ Pearson's correlation } & \multicolumn{3}{|c|}{ Parent-child } & \multicolumn{3}{|c|}{ Mother-child } & \multicolumn{3}{|c|}{ Father-child } \\
\hline & $\mathbf{N}$ & CC & $95 \% \mathrm{Cl}$ & $\mathbf{N}$ & CC & $95 \% \mathrm{Cl}$ & $\mathbf{N}$ & CC & $95 \% \mathrm{Cl}$ \\
\hline Adolescent algorithm for parents & 1786 & 0.13 & 0.09 to 0.18 & 1570 & 0.14 & 0.10 to 0.19 & 216 & 0.04 & -0.09 to 0.18 \\
\hline Adult algorithm for parents & 1786 & 0.15 & 0.10 to 0.19 & 1570 & 0.16 & 0.11 to 0.20 & 216 & 0.06 & -0.08 to 0.19 \\
\hline Adjusted linear regression & $\mathbf{N}$ & RC & P value & $\mathbf{N}$ & RC & $P$ value & $\mathbf{N}$ & RC & $P$ value \\
\hline \multicolumn{10}{|l|}{ Sensitivity analysis } \\
\hline Adult algorithm for parents & 1783 & 0.13 & $<0.001$ & 1569 & 0.14 & $<0.001$ & 214 & 0.05 & 0.42 \\
\hline
\end{tabular}

Non-biological caregivers were excluded from these analyses $(n=16)$. Covariates in adjusted linear regression models include parent and child age, SEIFA disadvantage index, BMI and parent and child sex in models including both sexes.

BMI, body mass index; CC, correlation coefficients for Pearson; CHU9D, Cchild Hhealth Utility 9 Dimension; $n$, number of biological child-parent pairs with this measure; RC, estimated regression coefficient; SEIFA, Socio-Economic Indexes for Areas. 


\section{Strengths and limitations}

Strengths of the Child Health CheckPoint study include the large sample size and the multistage, clustered survey methodology that together allow for the derivation of accurate population estimates. This is the first study to provide HRQL concordance data between children's HRQL and the HRQL of their parents within a large cohort study. This is also the first study to identify the distribution of HRQL values using the CHU9D in pre-adolescent children and mid-life adults in Australia.

The population-based sampling of this cohort make the findings more relevant to the wider Australian child population than previous HRQL studies focused on children with particular health conditions. However, there was an under-representation of socioeconomically disadvantaged participants in both child and adult cohorts, which limits generalisability to disadvantaged groups. The over-representation of mothers in the adult cohort also reduces the precision around estimates for fathers.

The CheckPoint study targets multiple Australian health priorities. Measurements were collected on each child and their accompanying parent over a $3 \frac{1 / 2}{2}$ hour session. The length and the range of data that needed to be collected restricted the number of HRQL measures that could be used. HRQL scores are known to vary according to the particular instrument ${ }^{4}$ and, for preference-based measures, the scoring algorithm used. Therefore, the results in this study for HRQL distributions and for intergenerational concordance may well be dependent on the particular HRQL measures used.

\section{Findings in relation to other studies}

When comparing the HRQL results from this study to others it is important to consider the scoring algorithms applied in each study. Scoring algorithms for the CHU9D have been updated over time and differ between countries. As mentioned previously, the scoring algorithm applied in this analysis is the most recent Australian version. An Australian trial conducted on first graders (mean age 6.9 years) by Roberts et al used the CHU9D as an outcome measure with the same scoring algorithm as we used..$^{33}$ The mean score recorded for child HRQL was $0.8 .^{33}$ This is consistent with the CHU9D values presented in table 2. Chen et al compared HRQL (measured by the CHU9D) and BMI in Australian children (mean age 10.6 years). ${ }^{12}$ Their study population had a mean CHU9D score of 0.87, which is higher than the means in the CheckPoint; however, they applied an older adolescent scoring algorithm (G Chen, Research Fellow, Flinders University, 2016). HRQL results from the PedsQL non-preference-based measure are similar to previously reported values from child samples in the USA (mean score 79.6, SD 15.3). ${ }^{25}$

This is also the first study to report the distribution of CHU9D values in Australian adults, and as such we are unable to determine the comparability of this result. The CheckPoint mean of 0.78 for the adult AQoL-8D was lower than Richardson $e t$ als population norms, which reported a mean score of 0.86 for adults aged 35-44 years, ${ }^{26}$ and also lower than the mean adult score of 0.83 reported in a multicountry study. ${ }^{4}$ This difference could be attributed to our adult sample being made up entirely of parents, given that adults are known to experience a dip in life satisfaction in the years following childbirth. ${ }^{34}$ Future research could explore the influence child age has on parental quality of life.

The difference in CHU9D values when applying adult and adolescent scoring algorithms to participants' selfrated CHU9D health states are expected and are in line with previous research. ${ }^{35}$ This supports the fact that adolescents do have different health preferences to adults and that it is important that studies of adolescent health not only allow adolescents to provide their own responses to measures but also use adolescent values in any scoring applied to those measures.

\section{Meaning and implications for clinicians and policymakers}

Our results add to the child HRQL literature and provide estimates of the distribution of HRQL to be expected in Australian population samples. These results can be used for comparison purposes with other populations and samples.

A rapid review of the literature surrounding intergenerational concordance for HRQL found that this study was the first of its kind in both an Australian and international setting (Catchpool Major Report Master of Health Economics, Deakin University, 2016). The results in this study suggested that there is a small intergenerational concordance for HRQL when measured at the same point in time. This clear evidence of the association between child and parental (especially maternal) HRQL supports the need to consider all individuals as a member of a wider family ${ }^{6}$ and to consider the impact of child and adolescent health services on the HRQL of parents and the impact of adult health services on the HRQL of patients' children.

\section{Unanswered questions and future research}

This is the first study to explore the intergenerational concordance for HRQL at the population level. The strength of the concordance found in this study reflects the population-based nature of the sample; concordance may be higher in groups of children and/or parents with chronic health conditions, in which case it would be even more important to consider impacts of health services on the broader family. Concordance results are also limited to the single HRQL instrument used in both child and parent groups (CHU9D), and future research should examine how concordance varies across HRQL measures.

Acknowledgements This paper uses unit record data from Growing Up in Australia, the Longitudinal Study of Australian Children. The study is conducted in partnership between the Department of Social Services (DSS), the Australian Institute of Family Studies (AIFS) and the Australian Bureau of Statistics (ABS). Some study data were collected and managed using REDCap (Research Electronic Data Capture; www.project-redcap.org) electronic data capture tools. REDCap is a secure, web-based application designed to support data capture for research studies. The authors thank the LSAC and CheckPoint study participants, staff and students for their contributions. 
Contributors MC, LG, SAC and MW contributed to study conception and interpretation of results, drafted the initial manuscript, critically revised further drafts and approved the final manuscript as submitted. MC and ACG contributed interpretation of results, performed the statistical analysis, drafted the initial manuscript, critically revised further drafts and approved the final manuscript as submitted.

Funding This work was supported by the National Health and Medical Research Council (NHMRC) of Australia (Project Grants 1041352, 1109355), The Royal Children's Hospital Foundation (2014-241), the Murdoch Children's Research Institute (MCRI), The University of Melbourne and Financial Markets Foundation for Children (2014-055, 2016-310). The following authors were supported by the NHMRC: Early Career Fellowship (1035100) to LG; Senior Research Fellowship (1046518) to MW. MW was supported by Cure Kids, New Zealand. The MCRI administered the research grants for the study and provided infrastructural support (IT and biospecimen management) to its staff and the study, but played no role in the conduct or analysis of the trial. Research at the MCRI is supported by the Victorian Government's Operational Infrastructure Support Program. The Australian Department of Social Services played a role in study design; however, no other funding bodies had a role in the study design and conduct; data collection, management, analysis and interpretation; preparation, review or approval of the manuscript; and decision to submit the manuscript for publication.

Disclaimer The findings and views reported in this paper are those of the authors and should not be attributed to DSS, AIFS or the ABS.

Competing interests MW received support from Sandoz to present at a symposium outside the submitted work.

Patient consent for publication Not required.

Ethics approval The CheckPoint data collection protocol was approved by The Royal Children's Hospital (Melbourne, Australia) Human Research Ethics Committee (33225D) and The Australian Institute of Family Studies Ethics Committee (14-26).

Provenance and peer review Not commissioned; externally peer reviewed.

Data sharing statement The Longitudinal Study of Australian Children datasets and technical documents are available to researchers at no cost via a licence agreement. Data access requests are co-ordinated by the National Centre for Longitudinal Data. More information is available at https://dataverse.ada.edu.au/ dataverse/lsac.

Open access This is an open access article distributed in accordance with the Creative Commons Attribution Non Commercial (CC BY-NC 4.0) license, which permits others to distribute, remix, adapt, build upon this work non-commercially, and license their derivative works on different terms, provided the original work is properly cited, appropriate credit is given, any changes made indicated, and the use is non-commercial. See: http://creativecommons.org/licenses/by-nc/4.0/.

\section{REFERENCES}

1. Naughton MJ, Shumaker SA. The case for domains of function in quality of life assessment. Qual Life Res 2003;12(Suppl 1):73-80.

2. Drummond MF, Sculpher MJ, Claxton K, et al. Methods for the economic evaluation of health care programmes: Oxford University Press, 2015.

3. Brazier J, Ratcliffe J, Saloman J, et al. Measuring and valuing health benefits for economic evaluation: Oxford University Press, 2017.

4. Richardson J, Khan MA, lezzi A, et al. Comparing and explaining differences in the magnitude, content, and sensitivity of utilities predicted by the EQ-5D, SF-6D, HUI 3, 15D, QWB, and AQoL-8D multiattribute utility instruments. Med Decis Making 2015;35:276-91.

5. Quah SR. International Encyclopedia of Public Health: Elsevier Science, 2016.

6. Ungar WJ, Gerber A. Economic Evaluation in Child Health: Oxford University Press, 2009.

7. Chen G, Ratcliffe J. A Review of the development and application of generic multi-attribute utility instruments for paediatric populations. Pharmacoeconomics 2015;33:1013-28.

8. Stevens K. Developing a descriptive system for a new preferencebased measure of health-related quality of life for children. Qual Life Res 2009;18:1105-13.

9. Ratcliffe J, Huynh E, Chen G, et al. Valuing the Child Health Utility 9D: Using profile case best worst scaling methods to develop a new adolescent specific scoring algorithm. Soc Sci Med 2016;157:48-59.

10. Ratcliffe J, Stevens K, Flynn T, et al. An assessment of the construct validity of the CHU9D in the Australian adolescent general population. Qual Life Res 2012;21:717-25.
11. Chen G, Flynn T, Stevens K, et al. Assessing the Health-Related Quality of Life of Australian Adolescents: an empirical comparison of the Child Health Utility 9D and EQ-5D-Y Instruments. Value Health 2015;18:432-8.

12. Chen G, Ratcliffe J, Olds T, et al. BMI, health behaviors, and quality of life in children and adolescents: a school-based study. Pediatrics 2014;133:e868-74.

13. Furber G, Segal L. The validity of the Child Health Utility instrument (CHU9D) as a routine outcome measure for use in child and adolescent mental health services. Health Qual Life Outcomes 2015;13:22

14. Mugno D, Ruta L, D'Arrigo VG, et al. Impairment of quality of life in parents of children and adolescents with pervasive developmental disorder. Health Qual Life Outcomes 2007;5:22.

15. Hatzmann J, Heymans HS, Ferrer-i-Carbonell A, et al. Hidden consequences of success in pediatrics: parental healthrelated quality of life--results from the Care Project. Pediatrics 2008;122:e1030-8.

16. Hiscock H, Bayer J, Gold L, et al. Improving infant sleep and maternal mental health: a cluster randomised trial. Arch Dis Child 2007;92:952-8.

17. Sanson A, Johnstone R, The LSAC Research Consortium \& FaCS LSAC Project Team. Growing Up in Australia takes its first steps. Family Matters 2004;67:46-53.

18. Edwards B. Growing Up in Australia: The Longitudinal Study of Australian Children: Entering adolescence and becoming a young adult. Family Matters 2014:5.

19. Soloff C, Lawrence D, Johnstone R. LSAC technical paper number 1: sample design. Melbourne: Australian Institute of Family Studies, 2005.

20. Clifford SA, Davies S, Wake M. Child Health CheckPoint: Cohort summary and methodology of a physical health and biospecimen module for the Longitudinal Study of Australian Children. BMJ Open 2019;9(suppl 3):3-22.

21. Wake M, Clifford S, York E, et al. Introducing Growing Up in Australia's Child Health CheckPoint. Family Matters 2014;95:15-23.

22. Davies S, Clifford SA, Gillespie AN, et al. Longitudinal Study of Australian Children's Child Health CheckPoint Data Issues Paper December 2018. Melbourne: Murdoch Children's Research Institute, 2018.

23. Harris PA, Taylor R, Thielke R, et al. Research electronic data capture (REDCap)--a metadata-driven methodology and workflow process for providing translational research informatics support. J Biomed Inform 2009;42:377-81.

24. Varni JW, Seid M, Rode CA. The PedsQL: measurement model for the pediatric quality of life inventory. Med Care 1999;37:126-39.

25. Varni JW, Seid M, Kurtin PS. PedsQL 4.0: reliability and validity of the Pediatric Quality of Life Inventory version 4.0 generic core scales in healthy and patient populations. Med Care 2001;39:800-12.

26. Richardson J, Khan MA, Chen G, et al. Population norms and Australian profile using the Assessment of Quality of Life (AQoL) 8D utility instrument. 2012.

27. Richardson J, lezzi A, Khan MA, et al. Validity and reliability of the Assessment of Quality of Life (AQoL)-8D multi-attribute utility instrument. Patient 2014;7:85-96.

28. Kuczmarski RJ, Ogden CL, Grummer-Strawn LM, et al. CDC growth charts: United States. Adv Data 2000;314:1-27.

29. Olson LM, Lara M, Pat Frintner M. Measuring health status and quality of life for US children: relationship to race, ethnicity, and income status. Ambul Pediatr 2004;4(4 Suppl):377-86.

30. Australian Bureau of Statistics. Socio-Economic Indexes for Areas (SEIFA) -Technical Paper 2011. Canberra: Australian Bureau of Statistics, 2013.

31. Heeringa SG, West BT, Berglund PA. Applied survey data analysis: CRC Press, 2010.

32. Ellul S, Hiscock R, Mensah F, et al. Longitudinal Study of Australian Children's Child Health CheckPoint Technical Paper 1: weighting and non-response. Melbourne: Murdoch Children's Research Institute, 2018.

33. Roberts G, Quach J, Spencer-Smith M, et al. Academic outcomes 2 years after working memory training for children with low working memory: a randomized clinical trial. JAMA Pediatr 2016;170:e154568.

34. Qu L, de Vaus D. Life satisfaction across life course transitions. Journal of the Home Economics Institute of Australia 2015;22:15.

35. Ratcliffe J, Huynh E, Stevens K, et al. Nothing About Us Without Us? A Comparison of Adolescent and Adult Health-State Values for the Child Health Utility-9D Using Profile Case Best-Worst Scaling. Health Econ 2016;25:486-96. 